\section{AL-AZHAR Dental Journal}

F $\quad \mathrm{O} \quad \mathrm{r}$

$\mathrm{G} \quad \mathrm{i} \quad \mathrm{r}$
The Official Publication of The Faculty of Dental

Medicine For Girls,

Al-Azhar University Cairo, Egypt.

Print ISSN 2537-0308 • Online ISSN 2537-0316

ADJ-for Girls, Vol. 8, No. 3, July (2021) - PP. 451:459

\title{
The Potential Therapeutic Effect of Allogenic Bone Marrow Derived Mesenchymal Stem Cells on Carbimazole-Induced Hypothyroidism on Albino Rats' Tongue
}

\author{
Aya M. $\operatorname{Reyad}^{1 *}$, Heba A. Adawy ${ }^{2}$, Rehab A. Abdel Moneim ${ }^{3}$
}

Codex : 13/21.07

azhardentj@azhar.edu.eg

http://adjg.journals.ekb.eg

DOI: $10.21608 /$ adjg.2021.29218.1256

Oral Medicine \& Surgical Sciences (Oral Medicine, Oral \& Maxillofacial Surgery, Oral Pathology, Oral Biology)

\section{KEYWORDS}

Hypothyroidism, BM-MSCs,

Tongue papillae.

\begin{abstract}
Purpose: This study aimed to evaluate the potential effect of Bone Marrow Derived Mesenchymal Stem Cells (BM-MSCs) in reducing the complication of hypothyroidism induced by Carbimazole through histological examination of the tongue. Material and Methods: 30 adult male albino rats were used and divided into three groups: Control group (GI) consisted of 10 rats allowed to access freely balanced diet and freshwater supply throughout the experimental period ( 8 weeks), Hypothyroid group (GII) in which 10 rats received carbimazole dissolved in distilled water with a dose of $5 \mathrm{mg} / 250 \mathrm{~g}$ body weight/day by intra-gastric intubation for 5 weeks to induce hypothyroidism and BM-MSC group (GIII) in which 10 rats received carbimazole as in GII for 5 weeks and after confirmation of hypothyroidism received a single intravenous injection of BM-MSCs $\left(1 \times 10^{6}\right.$ cells $)$ through tail vein. All rats were sacrificed; tongue was dissected and processed for hematoxyline \& eosin and fluorescent microscopic evaluation. Results: Tongue papillae of GII showed marked morphological and histological alterations including hyperchromatic nuclei, multiple vacuolation among the cells and apparent decrease in the keartohyaline granules' content. Moreover, the connective tissue (C.T.) core appeared loose with inflammatory cell infiltrate and numerous congested blood vessels. On the other hand, the papillae in BM-MSCs treated groups showed apparent improvement in their architecture. Conclusion: Hypothyroidism has degenerative effects on tongue papillae. Administration of BM-MSCs reduces the deleterious effects of hypothyroidism on the papillae.
\end{abstract}

- Paper extracted from Master thesis titled "The Potential Therapeutic Effect of Bone marrow Derived Mesenchymal Stem Cells on Carbimazole-Induced Hypothyroidism on Albino Rats' Tongue."

1. Teaching assistant of oral biology, Oral Biology Department, Faculty of Oral and Dental Medicine, Future University in Egypt.

2. Professor of Oral Biology, Oral Biology Department, Faculty of Dental Medicine for Girls, Al -Azhar University, Cairo, Egypt

3. Professor of Oral Biology, Oral Biology Department, Faculty of Dentistry, Cairo University, Cairo, Egypt

* Corresponding author email: aya.reyad@hotmail.com 


\section{INTRODUCTION}

Thyroid gland is the main regulator of metabolism and ordinary function of body cells. Thyroid disorder is the second most common glandular disorder of the endocrine system which may intensely affect any tissue in the body including; the tongue tissues ${ }^{(1,2)}$. Hypothyroidism; a syndrome caused by thyroid hormones deficiency, is the most common thyroid disorder. It may result from thyroid disorder, from damage in mechanisms that control the development of thyroid hormones, or can arise as a result of complications during the treatment of hyperthyroidism ${ }^{(3)}$. The most common oral findings in hypothyroidism are macroglossia, delayed wound healing, dysgeusia, delayed eruption of teeth and altered tooth morphology ${ }^{(1)}$.

Carbimazole is known as an antithyroid medication used to prevent thyroid hormone synthesis and described medically to treat hyperthyroidism. Yet, when it is administrated in continuous dosage, it can block hormone synthesis and produce hypothyroidism ${ }^{(4)}$.Many studies reported that drugs inducing hypothyroidism can cause severe cellular damage in many tissues including; thyroid, liver, kidney, lung, pancreatic, gastric mucosa and salivary gland tissues ${ }^{(5-7)}$

The associations between oral diseases and systemic diseases, especially those causing hormonal disturbances as thyroid dysfunction and diabetes mellitus had received great attention in the last few decades ${ }^{(3)}$. Since hypothyroidism is a systemic illness, pathological changes is expected to develop in the tongue structure of patient with hypothyroidism.

Regenerative medicine is a research field which deals with replacing and regenerating human cells, tissues and organs to reestablish normal function. ${ }^{(8)}$ Stem cells play an important role in regenerative medicine because of their self-renewal capability and differentiation potential when cultured under specific conditions. Mesenchymal stem cells (MSCs) consider the most favorable resource for the cell-based therapy of inflammatory and degenerative diseases due to their immuno-modulatory properties, multi-lineage differentiation potential and pro-angiogenic characteristics. In addition, they have the ability to home into injured tissue and act as a reservoir of growth factors and regenerative molecules ${ }^{(9,10)}$

Among the MSCs, BM-MSCs is the most readily available source of stem cells as they are easy to harvest, able to home into specific tissues, differentiate into mesodermal, ectodermal and endodermal cells and stimulate a local repair response ${ }^{(11,12)}$.

From the fore mentioned; the present research aimed to assess the effect of experimentally induced hypothyroidism on the histological structure of adult rat tongue papillae and to highlight the possible ameliorating effect of BM-MSCs.

\section{MATERIAL AND METHODS}

\section{Material:}

Carbimazole: an anti-thyroid agent supplied as film coated tablets under the trade name "Carbimazole Tablets" (Chemical Industries Development, Giza, A.R.E.). Each tablet contained 5mg of carbimazole as an active ingredient.

Bone Marrow Derived Mesenchymal Stem Cells (BM-MSCs): allogenic BM-MSCs were isolated from tibia and fibula of albino rats. Samples of bone marrow were assembled and were carefully flushed using a syringe with $3 \mathrm{ml}$ L-DMEM supplemented with $10 \%$ FBS and 1\% L glutamine and antibiotics. To generate a single cell suspension, the same syringe was gently drawn up and down several times then the BM suspensions were cultured in polystyrene dishes. The non-adherent cells were removed from the culture by a series of washes in PBS, while the adherent cells were incubated and expanded as monolayer cultures in 5\% humidified 
$\mathrm{CO}_{2}$ at $37^{\circ} \mathrm{C}$ for $12-14$ days. At $80-90 \%$ confluence, the cells were dissociated using $0.25 \%$ trypsin and $0.01 \%$ EDTA then sub-cultured in new culture dishes, referring as first passage cultures. MSCs were identified in passage 3 by their adhesiveness, fibroblastic shape, expression of CD29 and CD90 but not CD45 and their power for osteogenic and adipogenic differentiation. Stem cells were then labelled with PKH26 dye which is a red fluorescent linker that binds to the cell membrane of stem cells. BM-MSCs isolation was done in Biochemistry Department, Faculty of Medicine, Cairo University.

\section{Animals:}

Thirty adult male albino rats weighing between 200-250 grams were used in this study. The rats were housed individually in the animal house of Faculty of Medicine, Cairo University. The animals were kept under controlled temperature, humidity, and dark-light cycle. They were allowed to access freely adequate balanced diet and fresh water throughout the experimental period. The experiment was done under supervision of specialized veterinarian since their housing till getting rid of the sacrificed bodies. The experiment was approved by Research Ethics Committee (REC18-037), Faculty of Dental Medicine for Girls, Al-Azhar University.

After one week acclimatization period, the animals were randomly divided into three groups (ten rats each):

- Control group (GI): allowed to access freely balanced diet and freshwater supply throughout the experimental period (8weeks).

- Hypothyroidism group (GII): rats received a single daily dose of carbimazole $(5 \mathrm{mg} / 250 \mathrm{~g}$ body weight/ day) dissolved in $3 \mathrm{ml}$ of distilled water and delivered by intragastric tube for 35 successive days (5 weeks). ${ }^{(7)}$

To confirm induction of hypothyroidism:

After five weeks from the beginning of carbimazole treatment, blood samples were drawn from retro orbital veins using capillary tubes. These samples were collected to measure the serum T3 and T4 levels ${ }^{(13)}$. Serum T3 and T4 concentrations were reduced in this group. The mean \pm SD serum T3 levels were $99 \pm 11.2 \mathrm{ng} / \mathrm{dl}$ (The reference value of the kit used for the T3 assay ranged between 205 and $269 \mathrm{ng} / \mathrm{dl})$. On the other hand, the mean serum levels of T4 were $4.08 \pm 0.6 \mu \mathrm{g} / \mathrm{dl}$ (The reference value of the kit used for the T4 assay ranged between 7.3 and $15 \mu \mathrm{g} / \mathrm{dl})$.

- BM-MSCs treated group (GIII): hypothyroidism was induced similar to group II. After confirmation of hypothyroidism, rats received a single dose injection of $\left(1 \times 10^{6}\right.$ cells $)$ of BMMSCs labelled with PKH26 fluorescent linker dye suspended in $(1 \mathrm{ml})$ phosphate buffer saline (PBS) per rat via tail vein ${ }^{(14)}$.

At the end of the experimental period (8 weeks); three weeks after the stem cells injection, the rats in all groups were killed with sodium thiopental overdose and their tongues were immediately dissected and prepared for light microscopic examination.

\section{Light microscopic examination}

The samples were fixed immediately in $10 \%$ formalin solution for $24 \mathrm{~h}$ before cutting to avoid tissue shrinkage or rolling. For filiform and fungiform papillae, the anterior two thirds of the tongue was cut sagittaly into two halves (along the median sulcus). For circumvallate papilla, the posterior one third was cut just anterior to the circumvallate papilla which was clearly seen macroscopically. The specimens were then properly washed under running water, dehydrated by transferring them in ascending grades of alcohol, cleared in xylene and then embedded in paraffin blocks. $4 \mu \mathrm{m}$ thick sections were obtained and mounted on glass slides ${ }^{(15)}$. The tongue sections were deparaffinized and rehydrated. The sections were finally stained by hematoxyline and eosin for routine histological evaluation. 


\section{RESULTS}

\section{A. Light microscopic results}

\section{Filiform papillae:}

- Control group (GI): The papillae exhibited uniform even distribution showing convex and concave sides. The tips of the papillae were pointed backward towards the base of the tongue with normal keratinized stratified squamous epithelium covering. The covering epithelium consisted of a basal layer, spinous layer, granular layer and the keratinous layer. The subepithelial connective tissue (C.T.) showed normal architecture and secondary C.T papillae.(Fig 1A)

- Hypothyroid group (GII): The papillae lost their characteristic flame-like appearance and appeared atrophied with rounded tips. They were covered by torn and separated keratin layer with hyperchromatic nuclei and multiple vacuolation among the cells. The granular layer showed apparent decrease in the keartohyaline granules' content. The C.T core was loose with inflammatory cell infiltrate and numerous congested blood vessels.(Fig 1B)

- BM-MSC treated group (GIII): the papillae appeared almost similar to those of control group with evenly distributed papillae regular in size, shape and orientation and normal keratinized stratified epithelium covering with few nuclear hyperchromatism. The C.T core was nearly normal with 1-2 secondary papillae, yet the C.T revealed few thick walled blood vessels.(Fig 1C)

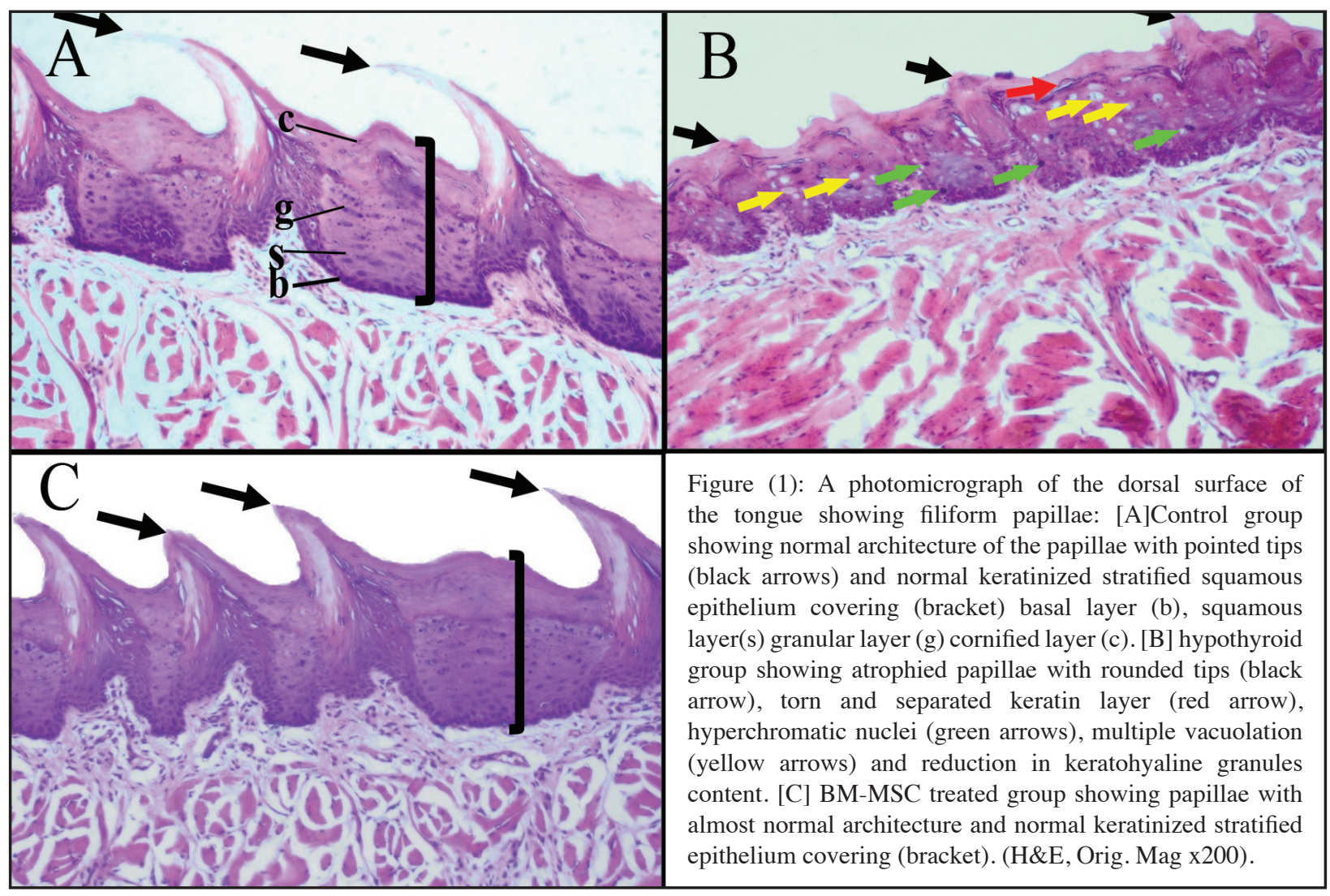




\section{Fungiform papillae:}

Control group (GI): the papillae assumed a mushroom shape and appeared elevated above the surface of the tongue. The papilla was covered by keratinized stratified squamous epithelium with a single well defined barrel shaped taste bud at its dorsal surface. Well-formed C.T core was demonstrated.(Fig 2A)

Hypothyroid group (GII): The papillae lost their characteristic appearance with narrow dorsal surface and atrophied taste buds. The epithelium showed scattered vacuolated cells and reduction in keratohyaline granules content. The lamina propria appeared loose with thick walled blood vessels.(Fig 2B)

BM-MSC treated group (GIII): restoration of the typical mushroom shaped papillae. The covering stratified squamous epithelium exhibited the regular histological structure and evidence of a single taste bud started to appear. However, few dilated and congested blood vessels together with numerous inflammatory cells were still evident in the lamina propria.(Fig 2C)

\section{Circumvallate papilla:}

Control group (GI): Single papilla appeared as inverted cone, not projecting above the surface of the tongue, surrounded by a well-developed deep trough and covered by a thin layer of keratinized stratified squamous epithelium. Numerous taste buds were present along the epithelial walls of the trough. Ducts of Von Ebner salivary glands were observed opening in the depth of the trough. A wellformed dense C.T. core was evident.(Fig 3A)

Hypothyroid group (GII): The papilla revealed detached keratin layer and widening of the trough. Taste buds were distorted and atrophied in certain areas yet, they were accumulated in several layers along the base of the trough. The lamina propria showed some inflammatory cells and areas of degeneration.(Fig 3B)

BM-MSC treated group (GIII): The papillae appeared almost similar to those of control group with slight widening in the trough.(Fig 3C)

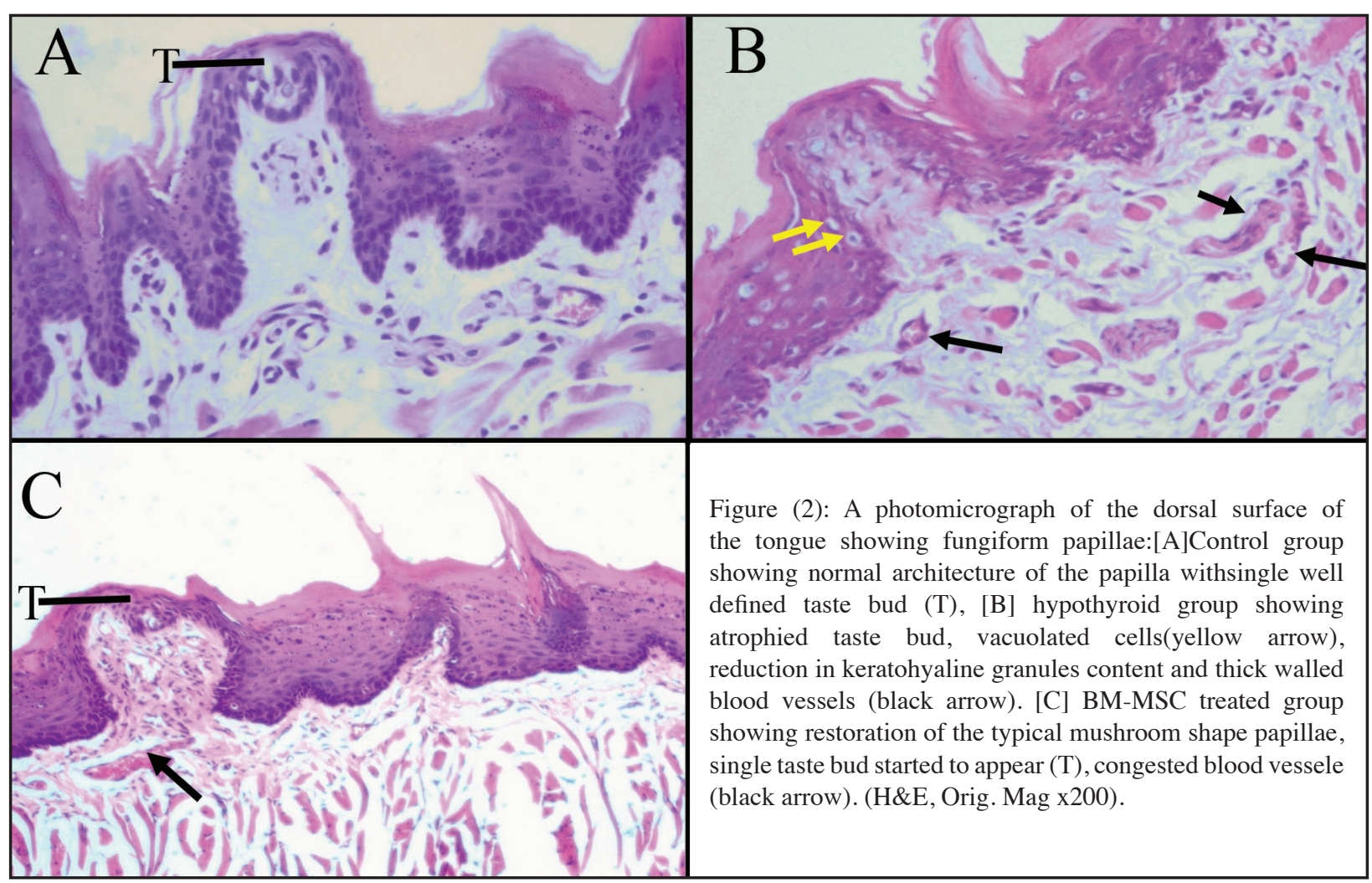




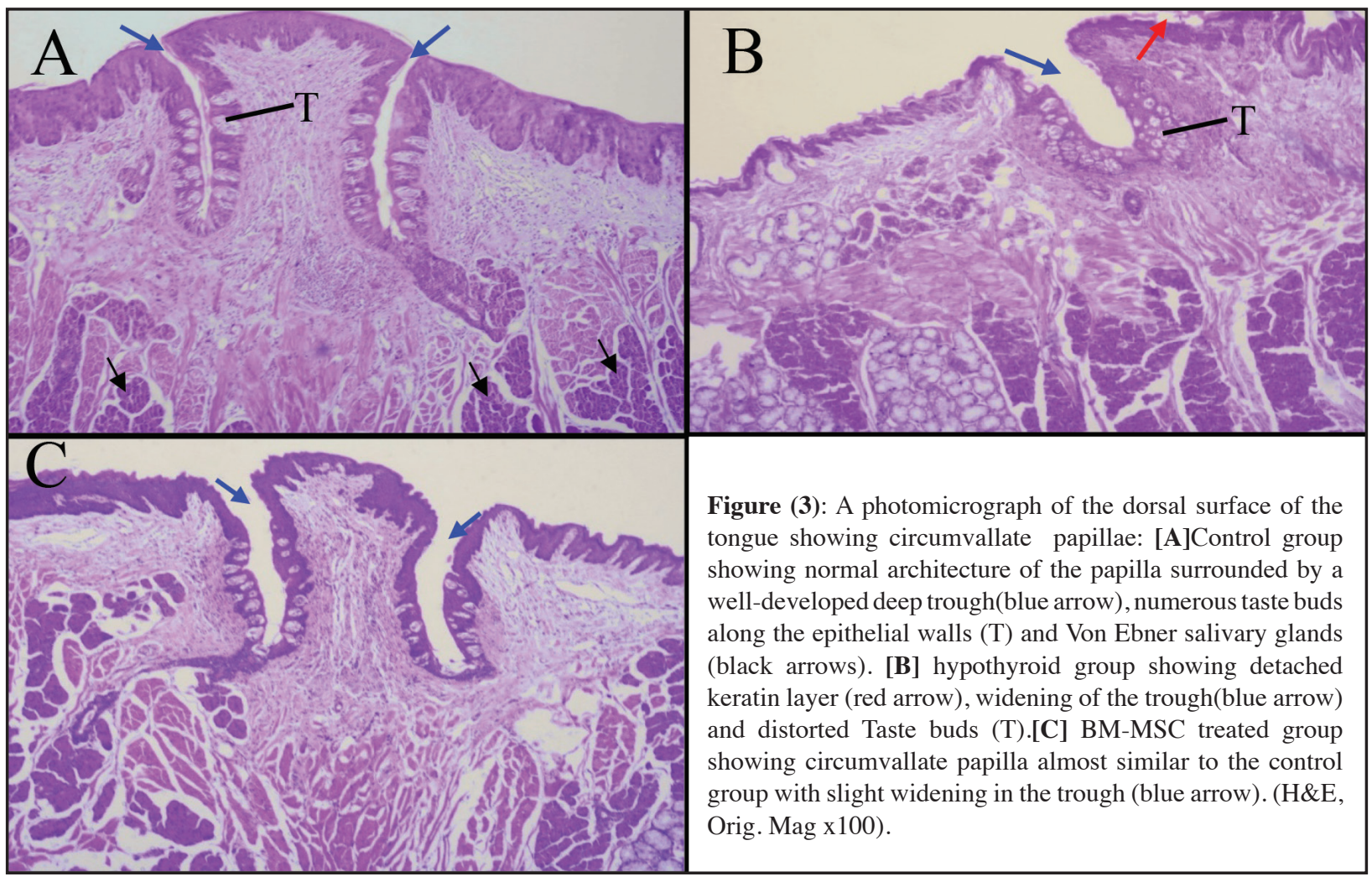

\section{B. Fluorescent microscopic result}

For homing assessment of MSCs, $5 \mu$ of paraffinembedded unstained sections were prepared and deparafinized, then examined using fluorescent microscopy (Sigma-Aldrich, Saint Louis, USA) to ensure homing of PKH26 labeled-MSC cells into the hypothyroid rat's tongue. PKH26 labeled

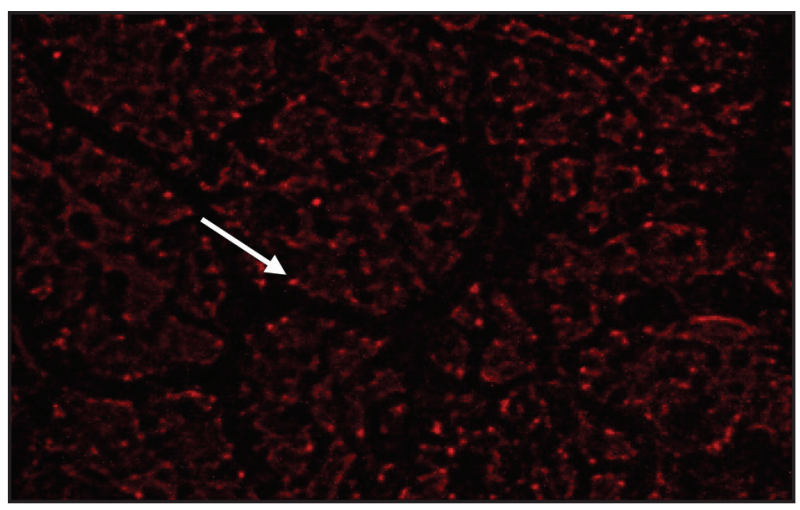

Figure (4): Photomicrograph of the dorsal surface of the tongue of BM-MSC group showing:red fluorescent PKH26 labeled cells in the tongue tissues 3 weeks following injection (white arrow). (PKH26, Original Mag. ×200) cells appeared as red dots on a dark background. Fluorescent microscopic examination was carried out at "The Tissue Culture Unit", Biochemistry Department, Faculty of Medicine, Cairo University, Egypt. (Fig 4)

\section{DISCUSSION}

Hypothyroidism; a syndrome caused by thyroid hormones deficiency, is one of the most common thyroid disorders in humans ${ }^{(2)}$. In this study, hypothyroidism was provoked by carbimazole; the most frequently and preferentially used drug in treatment of hyperthyroidism. The drug was given to the animals through a stomach tube so the dose could be accurately adjusted ${ }^{(5)}$. It was postulated that after absorption of carbimazole it is converted to the active form methimazole. Methimazole acts as a false substrate for thyroid peroxidise, thus reducing the production of the thyroid hormones. In our experimental model, development of hypothyroidism was confirmed by serum level of T3 and T4 hormones as there was a significant decrease in their expression. 
In the ongoing study, the rat was chosen as an animal model because it is one of the most widely used research animals whose physiological body functions are nearly similar to those of human being. In addition, they could be housed, bred and handled without difficulties. Adult male albino rats were chosen because they have more stable hormonal status than female rats ${ }^{(13)}$. Whereas, the tongue was selected for the investigation, as the tongue was considered a mirror that reflects the general health status ${ }^{(16)}$.

The associations between oral diseases and systemic diseases, especially those causing hormonal disturbances as thyroid dysfunction and diabetes mellitus had received great attention in the last few decades ${ }^{(17)}$. Since hypothyroidism is a systemic disorder it has been postulated that histological changes would affect the tongue tissues.

The current study was designed to provide a new research platform through exploring the effect of experimentally induced hypothyroidism on the adult rat tongue and assessing the possible ameliorating effect of BM-MSCs.

Light microscopic examination of H\&E stained sections of hypothyroid rat's tongue revealed several histological alterations in tongue papillae. These included presence of cellular and nuclear changes such as numerous vacuoles, hyperchromatism and binucleation among the keratinized stratified squamous epithelial cells. The obtained results were in agreement with previous study which reported vacuolation of the cytoplasm and darkly stained nuclei in the epithelium of renal cortex tubules of hypothyroid rats treated with methimazole ${ }^{(13)}$. The authors suggested that this effect was accompanied with oxidative stress, which was detected by the immunohistochemical stain. Other studies showed that hypothyroidism is associated with oxidative stresses due to insufficient anti-oxidant production ${ }^{(18-20)}$.

Moreover, the granular cell layer showed reduction in the keratohyaline granules content. This finding might be the result from the reduction in T3 hormone level; as this hormone has been shown to directly stimulate proliferation of epidermal keratinocytes ${ }^{(21)}$. Yet, the cornified cell layer showed separation of the keratin layer in hypothyroid rats. Previous authors mentioned that hypothyroidism affects the development of lamellar granules (Odland bodies) which are essential for the formation of a normal stratum corneum ${ }^{(22)}$.

In this study, the taste buds of group II were distorted and atrophied. Clinically, an altered taste perception was documented in patients with thyroid dysfunction ${ }^{(23,24)}$. It was mentioned that thyroid hormones have a well-known effect in the maturation of fungiform papilla. Thus, hypothyroidism could act as a damaging factor for the development of the papillae with subsequent decrease in taste perception ${ }^{(25)}$.

Examination of the specimens of group III revealed improvement in the architecture of the papillae. This was confirmed by reduction of vacuolation, uniform keratin layer and preservation of the taste buds and the characteristic shape of the papillae. It was reported that intravenous injection of BM-MSCs reduced the severity of induced stomatitis in rats receiving chemotherapy (26). Similarly, another study showed that BM-MSCs treatment reduced the damaging effects of diabetes on filiform and fungiform papillae of albino rats ${ }^{(14)}$. The improvement in BM-MSC treated group might be explained by several potential mechanisms. First; indirectly as systemic injection of BM-MSCs may cause regeneration of the thyroid gland ${ }^{(25)}$. Secondly; directly by homing of injected BM-MSCs in the tongue papillae and trans-differentiation into a diversity of cell types needed for tissue regeneration (14), this explanation was supported here by the fluorescent PKH26 labeled cells detection within the tissues of the tongue. Concomitantly, is the paracrine factors produced by BM-MSCs and the high expression of several pro-healing genes that ensure growth factors and cytokines production for a more prolonged period of time ${ }^{(14,27)}$. 


\section{CONCLUSION}

From the previously mentioned data; it was concluded that carbimazole induced hypothyroidism is associated with degenerative histological changes of the lingual papillae. Systemic injection of BMMSCs could be considered a successful approach that facilitated the regeneration of damaged tongue tissues. Thus, and due to the deteriorating effect of hypothyroidism on several tissues; further studies involving other oral tissues with alternative treatment protocols are recommended. It is also recommended to study the prophylactic effect of BMMSCs on hypothyroidism degenerative changes of different oral tissues.

\section{REFERENCES}

1. Kothiwale S, Panjwani V. Impact of thyroid hormone dysfunction on periodontal disease. J Sci Soc. 2016; 43: 34-7.

2. Chen C, Xie Z, Shen Y, Xia SF. The roles of thyroid and thyroid hormone in pancreas: physiology and pathology. IJE. 2018;2018:1-14.

3. Ayuob N. Histological and immunohistochemical study on the possible ameliorating effects of thymoquinone on the salivary glands of rats with experimentally induced hypothyroidism. EJH. 2016; 39:125-35.

4. Al-Shaikh MN, Abdul Wahab TA, Abdul Kareem SH. Hypothyroidism induced by carbimazole in diabetic mice and its Management Using Parsley and Eruca sativa oil. JPBS. 2014; 9: 24:7.

5. Shehata MR, Mohamed DA, El-Meligy MM, Bastwrous AE. The effect of maternal hypothyroidism on the postnatal development of the pituitary-thyroid axis in albino rats: a histological, morphometric, and immunohistochemical study. J Curr Med Res Pract. 2017; 2: 79-97.

6. Europa E, Valdivia V, Colin M, Casas C, Butr R. Methimazole-induced hypothyroidism causes cellular damage in the spleen, heart, liver, lung and kidney. Acta Histochemica. 2011; 113:1-5.

7. Ajayi A, Adelakun A, Akhigbe R. Gastric Mucosa Damage and Impairment of Secondary Immune Response in Dysthyroidism is Associated with TNF- $\alpha$ Expression. Int $\mathbf{J}$ Biol Med Res. 2018; 8: 6063-9.
8. Jain A, Bansal R. Applications of regenerative medicine in organ transplantation. J Pharm Bioall Sci. 2015; 7:188-94.

9. Hollenberg AN, Choi J, Serra M, Kotton DN. Regenerative Therapy for Hypothyroidism: Mechanisms and Possibilities. Mol Cell Endocrinol. 2017; 445: 35-41.

10. Requicha JF, Viegas CA, Albuquerque CM, Azevedo JM, Reis RL, Gomes ME. Effect of Anatomical Origin and Cell Passage Number on the Stemness and Osteogenic Differentiation Potential of Canine Adipose-Derived Stem Cells. SCRR. 2012; 8:1211-22.

11. Li M \& Ikehara. Bone-Marrow-Derived Mesenchymal Stem Cells for Organ Repair. Stem Cells Int. 2013;2013:1-8.

12. Musiał-Wysocka A, Kot M, Majka M. The Pros and Cons of Mesenchymal Stem Cell-Based Therapies. Cell transplant. 2019; 28: 801-12.

13. Khatab MM, Hulail M, Hegazy A, Mohammed HO.Study of the Toxic Effect of Methimazole on the Cortical Structure of Adult Male Albino Rats and the Ameliorated Effect of Thyroxin. Z.U.M.J. 2018; 24: 208-19.

14. Mohsen RO, Halawa AM, Hassan R. Role of bone marrowderived stem cells versus insulin on filiform and fungiform papillae of diabetic albino rats (light, fluorescent and scanning electron microscopic study). Acta Histochemica. 2019; 7: 812-22.

15. Bancroft JD, Stevens A, Layton C. Theory and practice of histological techniques 7th ed. Elsevier, Churchill Livingstone, New York, 2013.

16. El shawakh1 WO, El-Sawa AA, Shafik SK. Protective Role of Hony After Lead Toxicity on Albino Rat Lingual Papillae. ADJ. 2016; 41:214-19

17. Hanau KJ, Naoom ER, Mahammed HO. Cpitn in Iraqi Females with Thyroid Dysfunction. MDJ. 2012; 9: 99-106.

18. Ajayi AF, Adelakun AA, Akhigbe RE. Gastric Mucosa Damage and Impairment of Secondary Immune Response in Dysthyroidism is Associated with TNF- $\alpha$ Expression. Int J Biol Med Res.2017; 8:6063-69.

19. Chakrabarti SK, Ghosh1 S, Banerjee S, Mukherjee S, Chowdhury S. Oxidative stress in hypothyroid patients and the role of antioxidant supplementation. Indian $\mathrm{J}$ Endocr Metab. 2016; 20: 674-8.

20. Al-Naely AJ, Shattnan DT. Effect of Grape Seed Extract on Biochemical Factor and Histological Changes in Liver and the Kidney in Albino Rat Infected Hypo- Hyperthyroidism Induced Laboratory by Carbimazole and L-Thyroxine. JGPT. 2017; 9:174-81. 
21. Safer J. Thyroid Hormone and Wound Healing. J Thyroid Res. 2013; 2013:1-5.

22. Selim SA, Alazouny ZM. The effect of experimental hypothyroidism on the skin of adult male albino rats and the therapeutic role of topical triiodothyronine: a histological and immunohistochemical study. EJH. 2015; 38: 649-58.

23. Hassan GS, Zahran DH. Effects of Experimentally Induced Hyperthyroidism on Rat Tongue Mucosa: Histological and Ultrastructural Study. Life Sci J. 2019;16:127-34.

24. Baskoy K, Ay SA, Altundag A, Kurt O, Salihoglu M, Deniz F, et al. Is There Any Effect on Smell and Taste Functions with Levothyroxine Treatment in Subclinical Hypothyroidism?. PLOS ONE. 2016; 11:1-8.
25. Arafa MA, Gouda ZA, El-naseery NI, Abdel-Nour HM, Hanafy SM, Mohamed AF, et al. Bone Marrow-Derived Mesenchymal Stem Cells Ameliorate the Pancreatic Changes of Chemically Induced Hypothyroidism by Carbimazole in Male Rats. Cells Tissues Organs J. 2018; 206:144-56.

26. Serry A, Shinaishin S, ElHaddad K. Effect of mesenchymal stem cells injection on induced stomatitis in chemotherapy treated rats.J Am Sci. 2017;13:41-50.

27. Rashed FM, GabAllah OM,AbuAli SY, Shredah MT. The Effect of Using Bone Marrow Mesenchymal Stem Cells Versus Platelet Rich Plasma on the Healing of Induced Oral Ulcer in Albino Rats. Int J Stem Cells. 2019; 12:95106. 